David A. Kopriva

\title{
Implementing Spectral Methods for Partial Differential Equations
}

Algorithms for Scientists and Engineers

Springer 


\section{Contents}

Preface $\ldots \ldots \ldots \ldots \ldots \ldots \ldots \ldots \ldots \ldots \ldots$ vii

\section{Part I Approximating Functions, Derivatives and Integrals}

1 Spectral Approximation . . . . . . . . . . . . . . 3

1.1 Preamble: Series Solution of PDEs . . . . . . . . . . . . . 3

1.2 The Fourier Basis Functions and Fourier Series . . . . . . . . . . 4

1.3 Series Truncation . . . . . . . . . . . . . . . 6

1.4 Modal vs. Nodal Approximation . . . . . . . . . . . . . 11

1.5 Discrete Orthogonality and Quadrature . . . . . . . . . . . . . 11

1.6 Fourier Interpolation . . . . . . . . . . . . . . . . . . . 14

1.6.1 Direct Computation of the Fourier Interpolation . . . . . 17

1.6.2 Error of the Fourier Interpolation . . . . . . . . . . 19

1.7 The Derivative of the Fourier Interpolant . . . . . . . . . . 21

1.8 Polynomial Basis Functions . . . . . . . . . . . . . . 23

1.8.1 The Legendre Polynomials . . . . . . . . . . . . . 24

1.8.2 The Chebyshev Polynomials . . . . . . . . . 25

1.9 Polynomial Series . . . . . . . . . . . . . . . . 26

1.10 Polynomial Series Truncation . . . . . . . . . . . . 28

1.10.1 Derivatives of Truncated Series . . . . . . . . . 30

1.11 Polynomial Quadrature . . . . . . . . . . . . . 31

1.12 Orthogonal Polynomial Interpolation . . . . . . . . . . 35

$2 \quad$ Algorithms for Periodic Functions . . . . . . . . . . . . . . . . . . 39

2.1 How to Compute the Discrete Fourier Transform . . . . . . . . . . 39

2.1.1 Fourier Transforms of Complex Sequences . . . . . . . . . 40

2.1.2 Fourier Transforms of Real Sequences . . . . . . . . . 43

2.1.3 The Fourier Transform in Two Space Variables . . . . . . . 48

2.2 The Real Fourier Transform . . . . . . . . . . . . . . . . 50

2.3 How to Evaluate the Fourier Interpolation Derivative by FFT . . . 53

2.4 How to Compute Derivatives by Matrix Multiplication . . . . . . . 54

3 Algorithms for Non-Periodic Functions . . . . . . . . . . . . . . . 59

3.1 How to Compute the Legendre and Chebyshev Polynomials . . . 59

3.2 How to Compute the Gauss Quadrature Nodes and Weights . . . 62

3.2 .1 Legendre Gauss Quadrature . . . . . . . . . . . . . . 62

3.2.2 Legendre Gauss-Lobatto Quadrature . . . . . . . . . 64

3.2.3 Chebyshev Gauss Quadratures . . . . . . . . . . 67

3.3 How to Evaluate Chebyshev Interpolants via the FFT . . . . . 67

3.3.1 The Fast Chebyshev Transform . . . . . . . . . . 68

3.4 How to Evaluate Polynomial Interpolants in Lagrange Form . . . . 73 
3.5 How to Evaluate Polynomial Derivatives . . . . . . . . . . . 78

3.5.1 Direct Evaluation of the Derivative . . . . . . . . . . . 79

3.5.2 Evaluation of Derivatives by Matrix Multiplication . . . . . 81

3.5.3 Even-Odd Decomposition . . . . . . . . . . . . 82

3.5.4 Evaluation by Transform Methods . . . . . . . . . . . . . 84

3.5.5 Performance of Various Polynomial Derivative Algorithms 84

\section{Part II Approximating Solutions of PDEs}

4 Survey of Spectral Approximations . . . . . . . . . . . . . . . . . 91

4.1 The Fourier Collocation Method . . . . . . . . . . . . . . . . . . 94

4.1.1 How to Implement the Fourier Collocation Method . . . . 96

4.1 .2 Benchmark Solution . . . . . . . . . . . . . . . . . . 99

4.2 The Fourier Galerkin Method . . . . . . . . . . . . . . . . . 101

4.2.1 How to Implement the Fourier Galerkin Method . . . . . . 103

4.2.2 Benchmark Solution . . . . . . . . . . . . . . . 106

4.3 Nonlinear and Product Terms . . . . . . . . . . . . . . . 107

4.3.1 The Galerkin Approximation . . . . . . . . . . . . . 107

4.3.2 How to Compute the Convolution Sum . . . . . . . . . 109

4.3.3 The Collocation Approximation . . . . . . . . . . . . . 112

4.4 Polynomial Collocation Methods . . . . . . . . . . . . . 115

4.4.1 Approximation of the Diffusion Equation . . . . . . . . . . 115

4.4.2 How to Implement the Methods . . . . . . . . . . . . . . 117

4.4.3 Benchmark Solution . . . . . . . . . . . . . . . 119

4.4.4 Approximation of Scalar Advection . . . . . . . . . 120

4.5 The Legendre Galerkin Method . . . . . . . . . . . . . . . . . 123

4.5.1 How to Implement the Method . . . . . . . . . . . . . . 127

4.6 The Nodal Continuous Galerkin Method . . . . . . . . . . . . . . 129

4.6.1 How to Implement the Method . . . . . . . . . . . . 133

4.6.2 Benchmark Solution . . . . . . . . . . . . 134

4.7 The Nodal Discontinuous Galerkin Method . . . . . . . . . . . . 134

4.7.1 How to Implement the Method . . . . . . . . . . 138

4.7.2 Benchmark Solution . . . . . . . . . . . . . . . 143

4.8 Summary and Some Broad Generalizations . . . . . . . . . . . . 144

5 Spectral Approximation on the Square . . . . . . . . . . . . . . . 149

5.1 Approximation of Functions in Multiple Space Dimensions . . . 149

5.2 Potential Problems on the Square . . . . . . . . . . . 151

5.2.1 The Collocation Approximation . . . . . . . . . . . . 152

5.2.2 The Nodal Galerkin Approximation . . . . . . . . . . . . . 173

5.3 Approximation of Time Dependent Advection-Diffusion . . . . . . 188

5.3.1 The Collocation Approximation . . . . . . . . . . . . . 188

5.3 .2 The Nodal Galerkin Approximation . . . . . . . . . . . . 189

5.3 .3 Time Integration . . . . . . . . . . . . . . . . . 191

5.3.4 How to Implement the Approximations . . . . . . . . . . 193

5.3.5 Benchmark Solution: Advection and Diffusion of a Spot

in a Uniform Flow . . . . . . . . . . . . . . . . 200 
5.4 Approximation of Wave Propagation Problems . . . . . . . . . 202

5.4.1 The Nodal Discontinuous Galerkin Approximation . . . . . 204

5.4.2 How to Implement the Nodal Discontinuous Galerkin

Approximation . . . . . . . . . . . . . 212

5.4.3 Benchmark Solution: Plane Wave Propagation . . . . . . 216

5.4.4 Benchmark Solution: Propagation of a Circular Sound Wave 217

6 Transformation Methods from Square to Non-Square Geometries . . 223

6.1 Mappings and Coordinate Transformations . . . . . . . . . . 223

6.1.1 Mapping a Straight Sided Quadrilateral . . . . . . . . . 224

6.1.2 How to Approximate Curved Boundaries . . . . . . . . . 225

6.1.3 How to Map the Reference Square to a Curved-Sided

Quadrilateral . . . . . . . . . . . . . . . . . . 229

6.2 Transformation of Equations under Mappings . . . . . . . 231

6.2 .1 Two-Dimensional Forms . . . . . . . . . . . . . . 238

6.3 How to Approximate the Metric Terms . . . . . . . . . . . 240

6.4 How to Compute the Metric Terms . . . . . . . . . . . . . 242

7 Spectral Methods in Non-Square Geometries . . . . . . . . . . . 247

7.1 Steady Potentials in a Quadrilateral Domain . . . . . . . . . 247

7.1.1 The Collocation Approximation . . . . . . . . . . . . . 247

7.1.2 The Nodal Galerkin Approximation . . . . . . . . . . 252

7.1.3 Solution of the Linear Systems . . . . . . . . . . . . 254

7.1.4 Benchmark Solution: Potential in Non-Square Domains . . 259

7.1.5 Benchmark Solution: Incompressible Flow over a Circular

Obstacle . . . . . . . . . . . . . . . . . 261

7.2 Steady Potentials in an Annulus . . . . . . . . . . . . 264

7.2.1 Benchmark Solution: Potential in an Annulus with a Source 271

7.3 Advection and Diffusion in Quadrilateral Domains . . . . . . . 272

7.3.1 Transformation of the Advection-Diffusion Equation . . . . 272

7.3.2 The Collocation Approximation . . . . . . . . . . . . . 273

7.3.3 The Nodal Galerkin Approximation . . . . . . . . . . . 274

7.3 .4 How to Implement the Approximations . . . . . . . . . 275

7.3.5 Benchmark Solution: Advection and Diffusion in a Non-Square Geometry . . . . . . . . . . . . . 276

7.3.6 Benchmark Solution: Advection and Diffusion of a Pollutant in a Curved Channel . . . . . . . . . . 277

7.4 Conservation Laws in Quadrilateral Domains . . . . . . . . . . 279

7.4.1 The Nodal Discontinuous Galerkin Approximation . . . . 280

7.4.2 How to Implement the Nodal Discontinuous Galerkin Approximation . . . . . . . . . . . . . . . 282

7.4.3 Benchmark Solution: Acoustic Scattering off a Cylinder . . 285

8 Spectral Element Methods . . . . . . . . . . . . . . . . . . . . . 293

8.1 Spectral Element Methods in One Space Dimension . . . . . . . 296

8.1.1 The Continuous Galerkin Spectral Element Method . . . . 297 
8.1.2 How to Implement the Continuous Galerkin Spectral Element Method . . . . . . . . . . . . . . 301

8.1.3 Benchmark Solution: Cooling of a Temperature Spot . . . . 305

8.1.4 The Discontinuous Galerkin Spectral Element Method . . . 308

8.1.5 How to Implement the Discontinuous Galerkin Spectral Element Method . . . . . . . . . . . . . . . 310

8.1.6 Benchmark Solution: Wave Propagation and Reflection . . 315

8.2 The Two-Dimensional Mesh and Its Specification . . . . . . . . . 317

8.2.1 How to Construct a Two-Dimensional Mesh . . . . . . . . 321

8.2.2 Benchmark Solution: A Spectral Element Mesh for a Disk . 326

8.3 The Spectral Element Method in Two Space Dimensions . . . . . . 326

8.3.1 How to Implement the Spectral Element Method . . . . . . 331

8.3.2 Benchmark Solution: Steady Temperatures in a Long Cylindrical Rod . . . . . . . . . . . . . . . . 340

8.4 The Discontinuous Galerkin Spectral Element Method . . . . . . . 341

8.4.1 How to Implement the Discontinuous Galerkin Spectral Element Method . . . . . . . . . . . . . . . . 343

8.4.2 Benchmark Solution: Propagation of a Circular Wave in a Circular Domain . . . . . . . . . . . . . . . 344

8.4.3 Benchmark Solution: Transmission and Reflection from a Material Interface . . . . . . . . . . . . . . 347

A Pseudocode Conventions . . . . . . . . . . . . 355

B Floating Point Arithmetic .................... . . 359

C Basic Linear Algebra Subroutines (BLAS) . . . . . . . . . . . . . . 361

D Linear Solvers . . . . . . . . . . . . . . . . . . . . . . 363

D.1 Direct Solvers . . . . . . . . . . . . . . . 363

D.1.1 Tri-Diagonal Solver . . . . . . . . . . . . . 363

D.1.2 LU Factorization . . . . . . . . . . . . . . . . . . 364

D.2 Iterative Solvers . . . . . . . . . . . . . . . 368

E Data Structures . . . . . . . . . . . . . . . . . 373

E.1 Linked Lists . . . . . . . . . . . . . . . . . . . . . . . . 373

E.1.1 Example: Elements that Share a Node . . . . . . . . . . 376

E.2 Hash Tables . . . . . . . . . . . . . . . . . . . . . . 377

E.2.1 Example: Avoiding Duplicate Edges in a Mesh . . . . . . 381

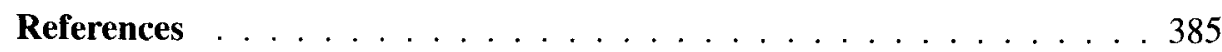

Index of Algorithms . . . . . . . . . . . . . . . . . . 387

Subject Index . . . . . . . . . . . . . . . . . . . . . . . . 389 